\title{
A simple method for deriving the uniform field MT responses in auroral zones
}

\author{
Alan G. Jones and Jessica Spratt* \\ Geological Survey of Canada, 615 Booth St., Ottawa, Ontario, K1M 0E9 Canada \\ (Received December 29, 2000; Revised October 2, 2001; Accepted November 3, 2001)
}

\begin{abstract}
Source field effects in magnetotelluric data acquired at high geomagnetic latitudes can result in erroneous interpretations of Earth conductivity structure deep within the mantle. This paper describes a simple technique most appropriate for a region that is dominantly one-dimensional (1-D) and uses the vertical magnetic field variations for identifying intervals of likely low contamination by non-uniform sources. Times are chosen when the variations stay within prescribed limits defined on the basis of a histogram of the variations for the whole recording interval. An example is given showing application of the method for data from a site under the auroral oval at a time when solar activity was at its lowest for the last solar cycle. A model derived from the responses obtained by processing all available data implies a decrease in resistivity at about $350 \mathrm{~km}$ to about $100 \Omega . \mathrm{m}$. In contrast, the model obtained from low activity interval responses shows a less rapid decrease in resistivity, without a change at around the 410 $\mathrm{km}$ phase boundary. The responses obtained from all data can be explained by the influence of a source with an average wavelength of $3,000 \mathrm{~km}$.
\end{abstract}

\section{Introduction}

\subsection{Brief historical perspective}

Conventional interpretation of crustal and upper mantle magnetotelluric (MT) response functions in the period range of $1-10^{4} \mathrm{~s}$ generally assumes that the external source field is incident as a plane wave on the surface of the Earth. Of course, this assumption is an approximation to the reality of time-varying three-dimensional (3-D) ionospheric and magnetospheric current systems that are sufficiently distant from the observation site so that the approximation is valid to within experimental error. This approximation was the subject of the first MT controversy, with Wait (1954) questioning its validity in his discussion of Cagniard's (1953) seminal paper, and their ensuing correspondence. Subsequently, Price (1962), and later Srivastava (1965), gave the pertinent theory for MT responses over a one-dimensional (1-D) Earth when the source field can be described by a single wavenumber $k$, given by $2 \pi / \lambda$ where $\lambda$ is the repetitive wavelength of the source. An example of the nature of the problem is illustrated in Fig. 1, where the MT responses over a 4-layered

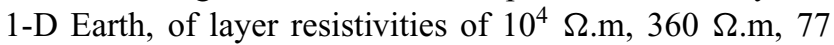
$\Omega . \mathrm{m}$ and $5 \Omega . \mathrm{m}$ and interface depths of $7 \mathrm{~km}, 46 \mathrm{~km}$ and $173 \mathrm{~km}$, are shown. The responses are for a uniform source field ( $\lambda=$ infinity, i.e., $k=0$ ), and source fields of wavelengths of $2,000 \mathrm{~km}, 1,000 \mathrm{~km}, 500 \mathrm{~km}$ and $200 \mathrm{~km}$. If the responses for a $1,000 \mathrm{~km}$ wavelength source are incorrectly interpreted as resulting from a plane-wave source, then the responses are best fit with a 4-layer 1-D model with layer

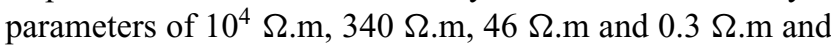

*Now at Department of Earth Sciences, William B. Heroy Geology Laboratory, Syracuse University, Syracuse, New York 13244, U.S.A.

Copy right (C) The Society of Geomagnetism and Earth, Planetary and Space Sciences (SGEPSS); The Seismological Society of Japan; The Volcanological Society of Japan; The Geodetic Society of Japan; The Japanese Society for Planetary Sciences. interface depths of $7 \mathrm{~km}, 48 \mathrm{~km}$ and $128 \mathrm{~km}$. The depth to the asthenosphere is underestimated by almost $50 \mathrm{~km}$ and its resistivity is underestimated by an order of magnitude. Accordingly, although the wavenumber parameter $k$ is independent of the other model parameters, its effect resembles that of making the conducting basal layer, which is the asthenosphere in most cases, more conducting and closer to the surface.

In reality, no source is repetitive in the space domain as required by a wavenumber description, and other source field geometries are listed in Table 1. A more realistic source geometry for sites influenced by the equatorial and auroral electrojets is a line current distribution (Hermance and Peltier, 1970) or a Gaussian current distribution (Peltier and Hermance, 1971). Hibbs and Jones (1976a, b) extended this work to an electrojet of arbitrary current distribution built from rectangular grids, Osipova et al. (1989) considered a two-dimensional (2-D) dipole source of limited extent, and Mareschal (1981), and later Viljanen et al. (1993, 1999) and Pirjola (1998), considered a realistic high latitude three-dimensional (3-D) current systems. Although the source fields themselves are 3-D, and Mareschal (1986) gave an excellent review for the interested reader, any 3-D current system can be represented exactly by an equivalent 2-D current distribution in a sheet (Akasofu, 1968). In almost all cases listed in Table 1, the Earth is assumed to have a 1-D resistivity-depth distribution.

Given the results displayed in Fig. 1, intuitively one might always expect a non-uniform source to lead to an underestimate of the plane-wave apparent resistivity. This is not the case for the effects due to field-aligned currents (Mareschal, 1981), or beyond approximately the half-width of an electrojet (Peltier and Hermance, 1971). Directly beneath a Gaussian electrojet the response is closely equal to that for a 
Table 1. Source field theory for a 1-D layered Earth model.

\begin{tabular}{ll}
\hline \multicolumn{1}{c}{ Source field geometry } & \multicolumn{1}{c}{ Reference } \\
\hline Uniform & Tikhonov (1950), Cagniard (1953) \\
Linear & Dmitriev and Berdichevsky (1979) \\
Wavenumber (repetitive in space) & Price (1962), Srivastava (1965) \\
Line Current & Hermance and Peltier (1970) \\
Gaussian & Peltier and Hermance (1971) \\
Arbitrary & Hibbs and Jones (1976a, b) \\
Dipole & Osipova et al. (1989) \\
3-D current system & Mareschal (1981), Viljanen et al. (1993, 1999), Pirjola (1998) \\
Moving Gaussian & Hermance (1978) \\
\hline
\end{tabular}

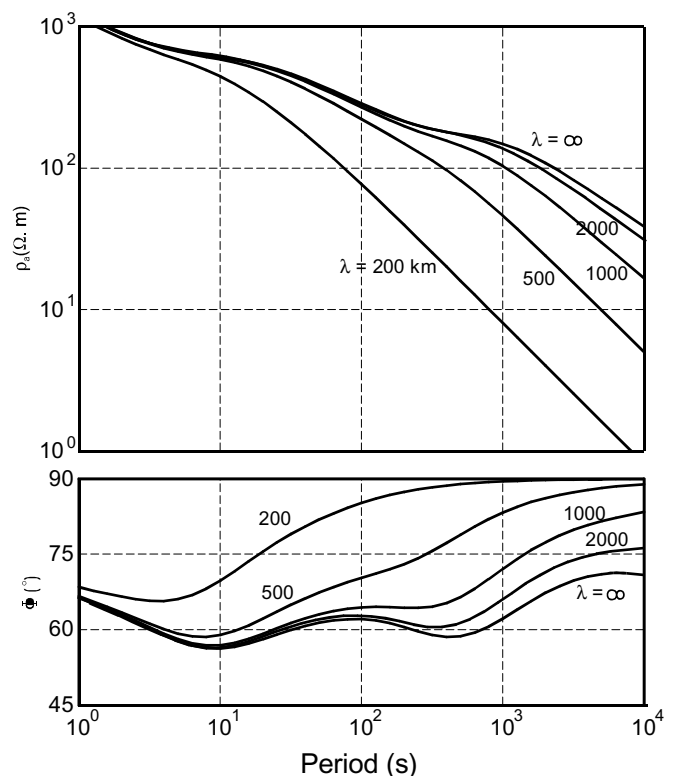

Fig. 1. Magnetotelluric responses over a 4-layer 1-D Earth, with layer resistivities of $10^{4} \Omega . \mathrm{m}, 360 \Omega . \mathrm{m}, 77 \Omega . \mathrm{m}$ and $5 \Omega . \mathrm{m}$ and interface depths at $7 \mathrm{~km}, 46 \mathrm{~km}$ and $173 \mathrm{~km}$, for a repetitive source with wavelengths of infinity, $2000 \mathrm{~km}, 1000 \mathrm{~km}, 500 \mathrm{~km}$ and $200 \mathrm{~km}$.

wavelength of four times the half-width of the source (Jones, 1980). However, at distances beyond the half-width from the centre the source field effect leads to an increase in apparent resistivity and a decrease in phase (Jones, 1980), and will thus lead to an overestimate of the depth to the asthenosphere. This result may appear to be counter-intuitive to those versed in wavenumber source descriptions. More complex and realistic 3-D sources show effects that are highly location and period-dependent, with effects that are unpredictable (Viljanen et al., 1999).

Implicit in all these representations is that the source is stationary in time and pulsates with a known frequency, and few approaches have used a moving source. However, a moving current source is the reality of the ionospheric and magnetospheric current systems, and Mareschal (1986) discusses the particular problem of the Harang discontinuity where the evening auroral eastward electrojet and the morning auroral westward electrojet meet. Hermance (1978) studied a laterally moving source with a $k$-wavenumber description and showed that the moving source has a larger "effective width," or smaller $k$. An exact representation requires using convolution integrals in both the space and time domains (Schmucker, 1970), and to date there have not been any modeling studies undertaken for a realistic source. One can conclude that the effects of a source field are temporally and spatially varying in a manner that cannot easily be predicted. Also, as discussed by Viljanen et al. (1993), one cannot assume that averaging over various source geometries will fortuitously yield the uniform source response.

\subsection{Theory}

The basis of the proposed method is most readily explained through use of Schmucker's (1970) inductive response function, $C(\omega, k)$ rather than the MT response function $Z(\omega, k) . C(\omega, k)$ is related to $Z(\omega, k)$ by:

$$
C(\omega, k)=\frac{1}{i \omega \mu_{0}} \cdot Z_{x y}(\omega, k)
$$

for a 1-D Earth. This inductive response function can also be derived using only the magnetic fields by either the Horizontal Spatial Gradient (HSG) method,

$$
C(\omega, k)=\frac{H_{z}(\omega, k)}{\frac{\partial H_{x}(\omega, k)}{\partial x}+\frac{\partial H_{y}(\omega, k)}{\partial y}}
$$

(Schmucker, 1970; Kuckes, 1973a, b; Schmucker and Weidelt, 1975; Jones, 1980) or from the relationship between the vertical and horizontal magnetic field components

$$
C(\omega, k)=\frac{H_{z}(\omega, k)}{i k\left(H_{x}(\omega, k)+H_{y}(\omega, k)\right)}
$$

(in this case $k$ must be known and in (3) is assumed to be the same in both horizontal directions). The latter can be rewritten as

$$
H_{z}(\omega, k)=i k C(\omega, k)\left(H_{x}(\omega, k)+H_{y}(\omega, k)\right)
$$

which emphasizes the point that there is no vertical magnetic field for plane wave induction $(k=0)$ in a 1-D Earth. This relationship is the basis of the method proposed herein. Although the above description is for the limited case of a repetitive source over a 1-D Earth, the essential point is the 
vertical field component is far more sensitive to non-uniform source field contributions than is the MT response.

The properties of $C(\omega, k)$ are discussed in Weidelt (1972), Schmucker and Weidelt (1975) and Jones (1980), and the latter detailed the restrictions on the inductive response function when expressed in the lag domain, $c(\tau, k)$. As discussed by Weidelt (1978) and Dmitriev and Berdichevsky (1979) (see also Mareschal, 1986), for a 1D Earth $C(\omega, k)$ can be interpreted as $C(\omega, 0)$ for a wider class of source structures than only a plane wave.

Those authors showed that the horizontal electric field can be expressed as a series of only even order space derivatives of the orthogonal horizontal magnetic field, whereas the vertical magnetic field is a series of only odd order derivatives. The direct consequence of this for MT is that $Z_{x y}(\omega, k)=Z_{x y}(\omega, 0)$ not only for a plane wave source $(k=0)$ but also for a source that varies linearly, or indeed with only odd order space derivatives, over a horizontal distance comparable to the inductive scale length. For the HSG method, then $C(\omega, k)=C(\omega, 0)$ when the fields are represented by the first space derivative and only even order higher space derivatives. This latter result was used by Jones (1980) in his HSG analysis, who fit the fields to second-order polynomials imposing a curl-free constraint. This dependence on derivatives may explain the poor HSG results obtained by Camfield (1981) who used a third-order polynomials and thereby implicitly included derivatives sensitive to non-plane wave source structure.

\section{Method}

Equation (4) shows that the transfer functions $\left(T_{x}, T_{y}\right)$ between the vertical component of the magnetic field and the two horizontal components, viz.,

$$
H_{z}(\omega, k)=T_{x}(\omega, k) H_{x}(\omega, k)+T_{y}(\omega, k) H_{y}(\omega, k)
$$

are equal to $i k C(\omega, k)$ for a source of wavenumber $k$ over a 1-D Earth. Figure 2 displays the modulus of this transfer function, $|k C(\omega, k)|$, for the same 1-D Earth model and same source wavelengths as Fig. 1. The figure demonstrates convincingly that the vertical field is far more sensitive to a non-uniform source field than is the MT response (Fig. 1), and that this sensitivity increases significantly with increasing period. Indeed, for highly non-uniform sources (200 $\mathrm{km}$ wavelength) at even moderately short periods (100 s) the response observed is purely due to the source geometry and no information about Earth structure can be obtained. This is analogous to the problems encountered in controlledsource EM methods where at low frequencies, and low is defined by the source geometry and the Earth's conductivity structure, the response is purely due to the source geometry. Mareschal (1981) shows the transfer functions for a more realistic geometry for an auroral source, emphasizing the point that they can become very large and indeed approach infinity as the horizontal magnetic field undergoes a reversal in polarity.

Accordingly, the amplitude of the vertical magnetic field is particularly sensitive to the presence of non-uniform source field contributions, leading to the proposed simple method for identifying times of likely low non-uniform

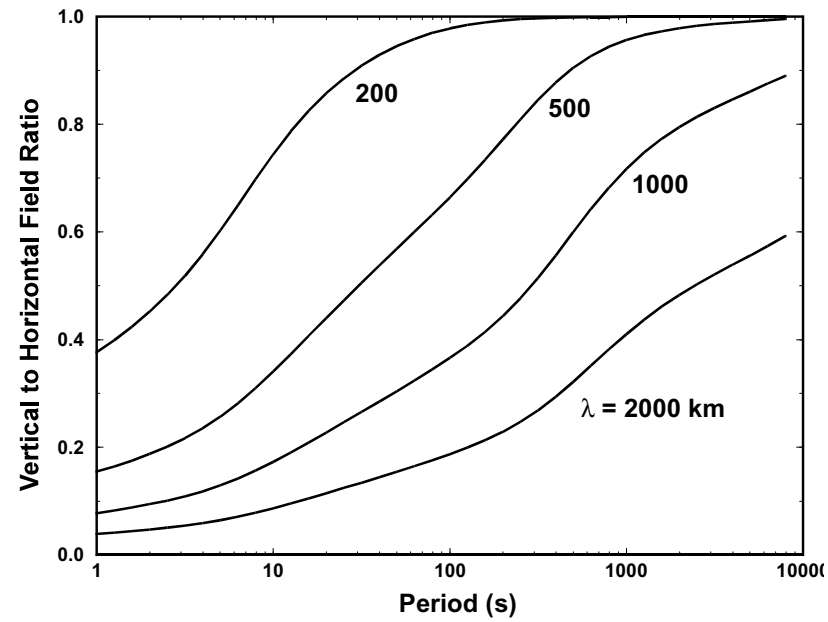

Fig. 2. Vertical field transfer function amplitude responses over a 4-layer

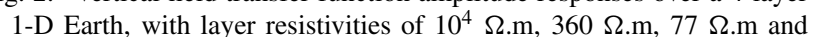
$5 \Omega . \mathrm{m}$ and interface depths at $7 \mathrm{~km}, 46 \mathrm{~km}$ and $173 \mathrm{~km}$, for a repetitive source with wavelengths of $2000 \mathrm{~km}, 1000 \mathrm{~km}, 500 \mathrm{~km}$ and $200 \mathrm{~km}$. For an infinite wavelength source (uniform field), the transfer function is zero.

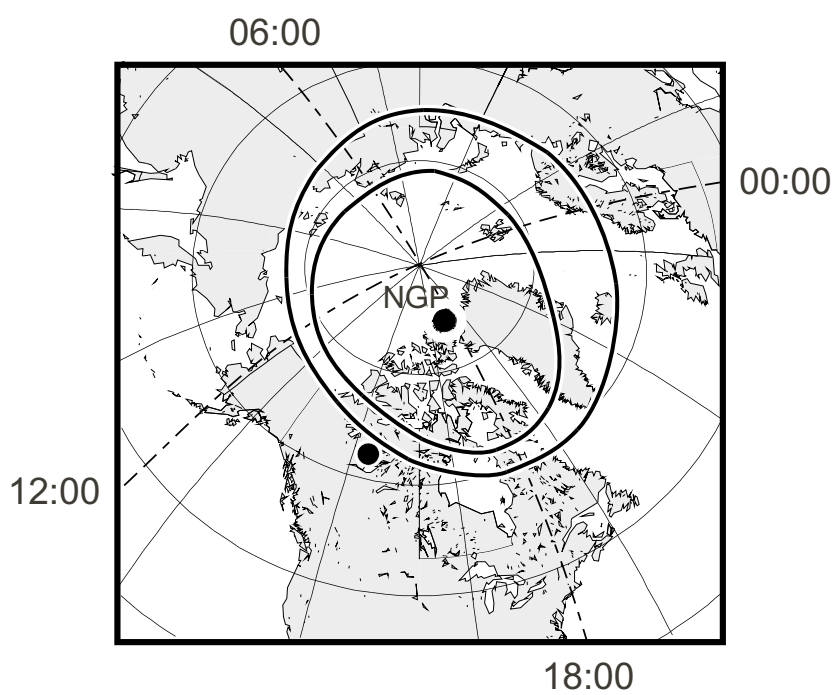

Fig. 3. Northern hemisphere showing the locations of the North Geomagnetic Pole (NGP), the quiet auroral electrojet, the site and relative magnetic local time. Modified after Pettersen (1992).

source field contribution: The data are scanned for time intervals of low vertical field amplitude, and only those intervals are used for calculation of the response functions.

\section{Example}

As an example of the application of this procedure, we use data acquired in northern Canada from a site located directly beneath the auroral oval at the southwestern edge of the Slave craton (Fig. 3). This particular site (site 107 in Jones and Ferguson, 2001) and this time interval were chosen specifically to demonstrate that problems can exist even during exceptionally low solar activity. Although we only demonstrate our approach on this single site, the methodology has been applied to all sites recorded in northern Canada 


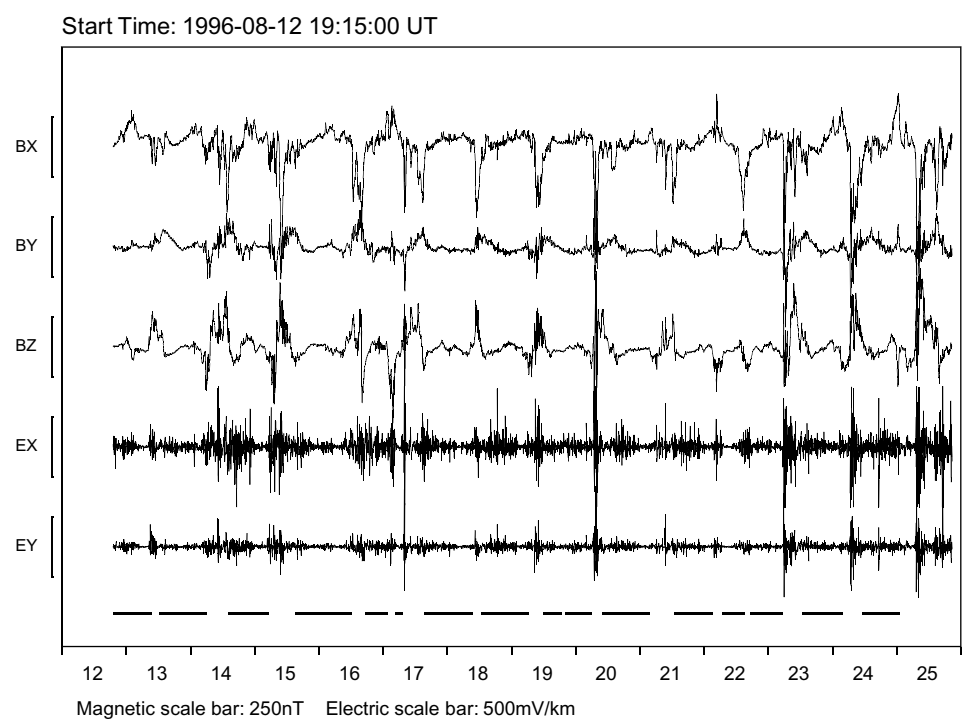

Fig. 4. Time series recorded at a site near Yellowknife, northern Canada, during August 1996. The bars indicate field variations of $250 \mathrm{nT}$ for the magnetic components, and $500 \mathrm{mV} / \mathrm{km}$ for the electric components. The horizontal bars show times when the vertical field component variations lay within the range -90 to $+60 \mathrm{nT}$.

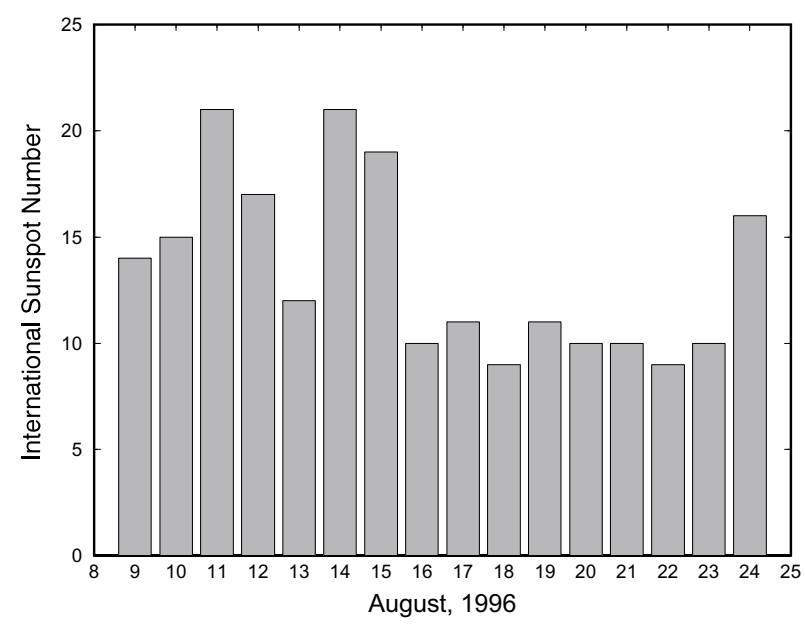

Fig. 5. Daily international sunspot numbers for the period just prior to and during acquisition of the data shown in Fig. 4. (Data obtained from the Sunspot Index Data Centre maintained by the Royal Observatory of Belgium.)

with equal success.

The site was occupied for two weeks, and the time series recorded are shown in Fig. 4. The MT system used was a Geological Survey of Canada LiMS (Long period Magnetotelluric System) with a sampling interval was $5 \mathrm{~s}$. The magnetic sensor was a 3-component low-noise ringcore fluxgate magnetometer designed by Narod and Bennest (1990), and the electrodes were low-noise non-polarizing $\mathrm{Pb}-\mathrm{PbCl}$ electrodes. Solar activity was low during the recording interval (1996-08-12 19:15:00 to 1996-08-25 20:55:10 UT); daily International Sunspot Numbers (ISN) varied between 12 and 21 from 1998-08-09 to 1996-08-24 (Fig. 5). (We show the ISN values for an overlapping interval preceding our MT data acquisition interval as solar wind streams of the Sun at typically $400 \mathrm{~km} / \mathrm{s}$, ranging from

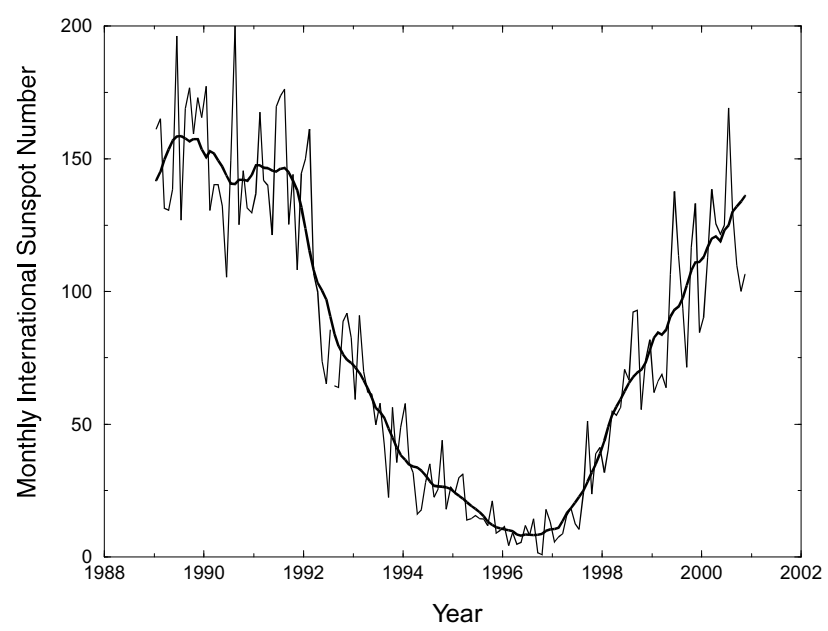

Fig. 6. Monthly international sunspot numbers for the last 11-year solar cycle. Note that the data shown in Fig. 4 were acquired during the minimum in the solar cycle.

$300-800 \mathrm{~km} / \mathrm{s}$, and thus takes three to four days to reach the surface of the Earth.) These values are close to solar minimum values for the last solar cycle (Fig. 6) testifying to the low solar activity during this time.

The region around the site is known to be laterally homogeneous, both geologically and geophysically (see Jones and Ferguson, 2001, for phase pseudosections - the site chosen, site 107 , is in the middle of the profile), and there are no strong conductivity contrasts in the crust or uppermost mantle. Although Swift's skew is magnitude-based and can be misleading in the presence of galvanic distortion, small values can only come from 1-D or 2-D regions (or from the centre of symmetric 3-D regions). For the responses derived from these data, Swift's skew is below 0.05 over the whole period range, and mostly below 0.02 . The maximum phase difference between the two off-diagonal elements is a supe- 
rior measure of dimensionality, and for these data is less than $\sim 10^{\circ}$ over the whole frequency range. Accordingly, given the predominant 1-D nature of the crust and upper mantle for this location, a uniform source field should induce very little vertical field component variation. Therefore, the $B z$ variations observed in Fig. 4 are almost entirely due to source field effects. There is a minor component due to structure, discussed below.

A histogram of the vertical field variations, from a baseline value of $60,410 \mathrm{nT}$, is shown in Fig. 7. The peak value is at $-24 \mathrm{nT}$, with a median value of $-17 \mathrm{nT}$, minimum and maximum variations of $-387 \mathrm{nT}$ and $+356 \mathrm{nT}$ respectively and $68 \%$ of the variations lie in the range -47 to $+30 \mathrm{nT}$. The small departure from zero for the median and peak values suggest a leveling error of the magnetometer of the order of $0.02^{\circ}$. Note that the distribution is both nonsymmetric and long-tailed, both of which suggest that con-

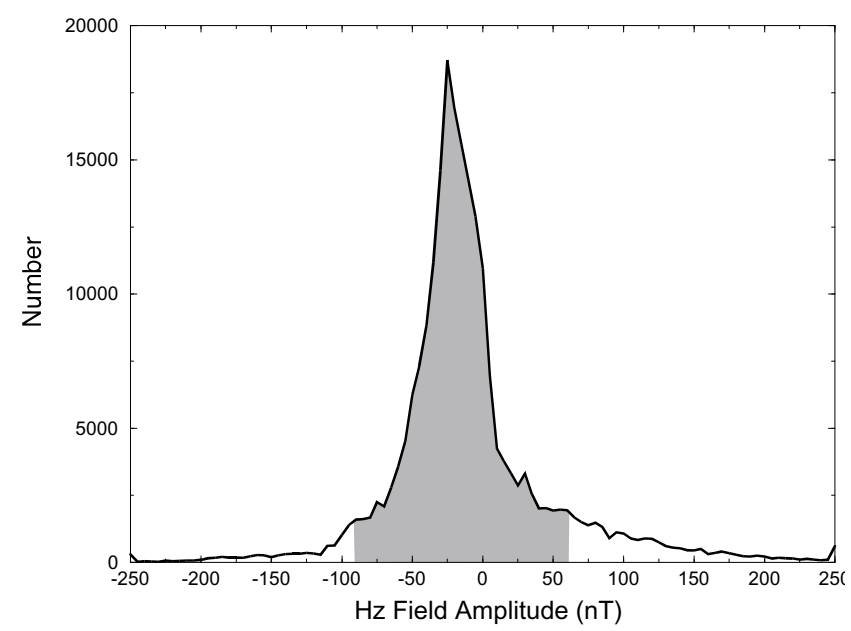

Fig. 7. Histogram of the vertical magnetic field variations shown in Fig. 4. The median and peak values are $-17 \mathrm{nT}$ and $-24 \mathrm{nT}$ respectively, and $68 \mathrm{nT}$. The shaded region shows the region between -90 to $+60 \mathrm{nT}$ (approx. 1.5 times one standard deviation). ventional spectral analysis assuming Gaussian distributed variables will fail.

One visual way of evaluating the nature of the contamination problem, manifest as time-varying responses, is to compute the complex attributes of the series, particularly the instantaneous ratio between the vertical magnetic field and the horizontal components of the magnetic field, using the analytical signals. Figure 8 shows the vertical component variations, $b_{z}(t)$, and the instantaneous amplitude of those variations $\left(b_{z} i(t)\right.$, middle time series, Fig. 8). The instantaneous amplitude is derived by numerical Hilbert transformation of the series (computed by fast Fourier transformation of $b_{z}(t)$, multiplying by $-i$, then inverse fast Fourier transformation, see Liu and Kosloff, 1981) to obtain the imaginary part of the analytical signal, then computation of the amplitude of the complex series. The instantaneous ratio (RATIO $i(t)$, bottom time series, Fig. 8) is derived from this instantaneous amplitude divided by the instantaneous amplitude of the horizontal components (given by $\left.\operatorname{sqrt}\left(b_{x} i(t) * b_{x} i(t)+b_{y} i(t) * b_{y} i(t)\right)\right)$. This instantaneous ratio varies from a low of 0.0 to a maximum of 8.6 with peak and median values of 0.50 and 0.56 respectively, and with $68 \%$ of the values lying in the interval 0.28 to 1.00 (Fig. 9). Note that this ratio correlates with the instantaneous amplitude of the vertical magnetic field, i.e., when the vertical field component is high, then the ratio of it to the horizontal field component is also high. This demonstrates that high amplitude vertical fields are associated with highly non-uniform sources, consistent with Eq. (4) and with our assumption that there is little vertical field response due to lateral structures.

Estimates of the MT transfer functions using all data were derived using the heuristic robust processing scheme originally proposed by Jones and Jödicke (1984) and modified by Jones (method 6 in Jones et al., 1989). The estimates were derived in a geographic coordinate system, after rotating the time series to correct for declination $\left(+26.7^{\circ}\right)$, and are shown in Fig. 10 (open symbols). Horizontal magnetic

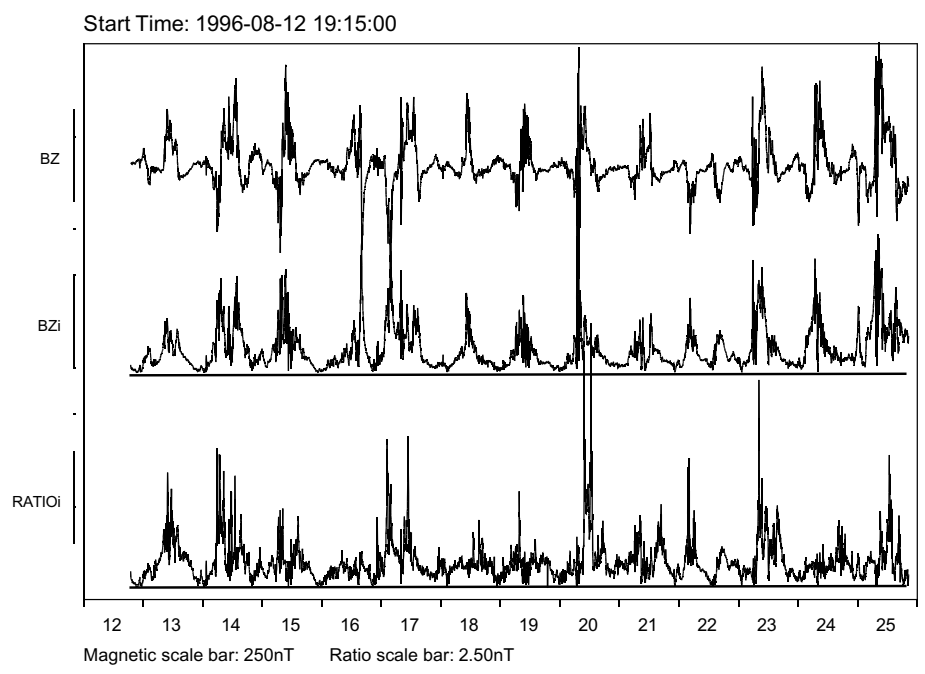

Fig. 8. Vertical field component variations (top series) plus its instantaneous amplitude (middle series). The ratio of this instantaneous amplitude to the instantaneous amplitude of the horizontal components is shown in the bottom series. 


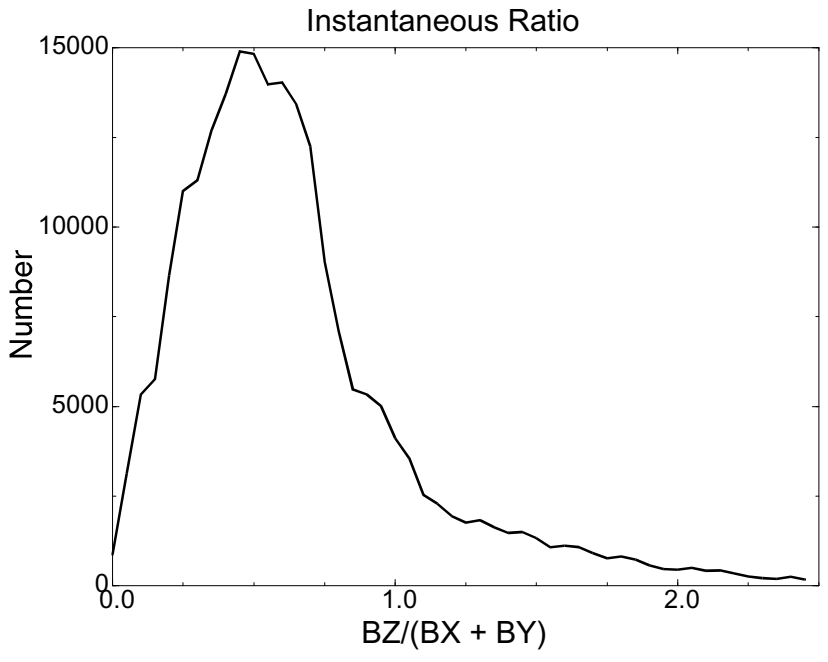

Fig. 9. Histogram of the instantaneous ratio between the vertical component and the horizontal components of the magnetic field. The peak and median values are 0.50 and 0.56 respectively, and $68 \%$ lie in the range 0.28 to 1.00
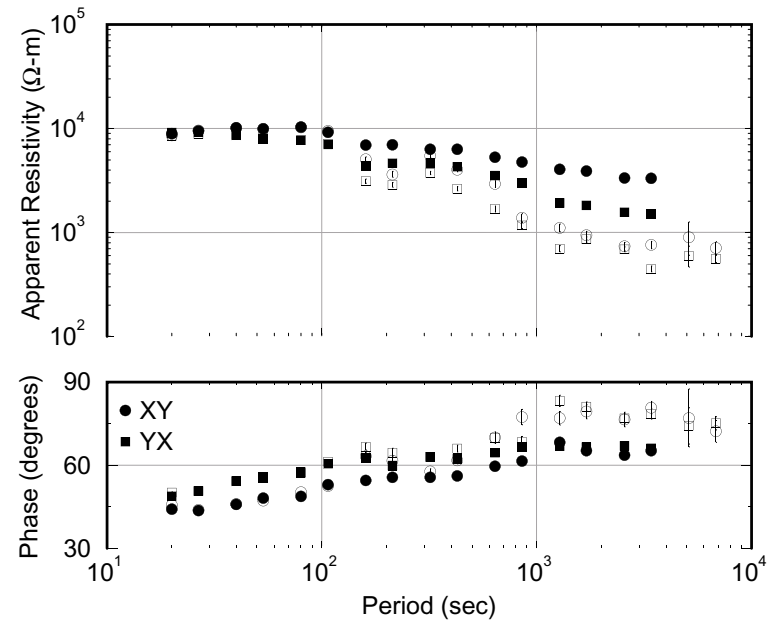

Fig. 10. Magnetotelluric response functions calculated in geographic coordinates using all data (open symbols) and using only those intervals when the vertical field variations lay within -90 to $+60 \mathrm{nT}$ (solid symbols). The circles denote the estimates for north-directed electric field and east-directed magnetic field (RhoXY and PhaXY), and the squares denote the complimentary east-directed electric field and south-directed magnetic field (RhoYX and PhaYX). Vertical bars indicate one sigma error estimates: where absent the errors are smaller than the symbol.

variations recorded simultaneously at a site approx. $65 \mathrm{~km}$ to the SW were used as remote reference series to avoid autopower bias (Gamble et al., 1979). These estimates visually appear reasonable, and a 1-D smooth model (Occam, Constable et al., 1987) that fits the geometric averaged response to within $5^{\circ}$, and equivalent for apparent resistivity, is shown in Fig. 11 (dashed line). The data were underfit on purpose to find the minimum smoothest structure that describes the majority of the response. It is possible to find 1-D smooth models that fit to within smaller error bounds (to $2^{\circ}$ in phase and equivalent in apparent resistivity); these models have the same features as the one shown in Fig. 11 but with more se-

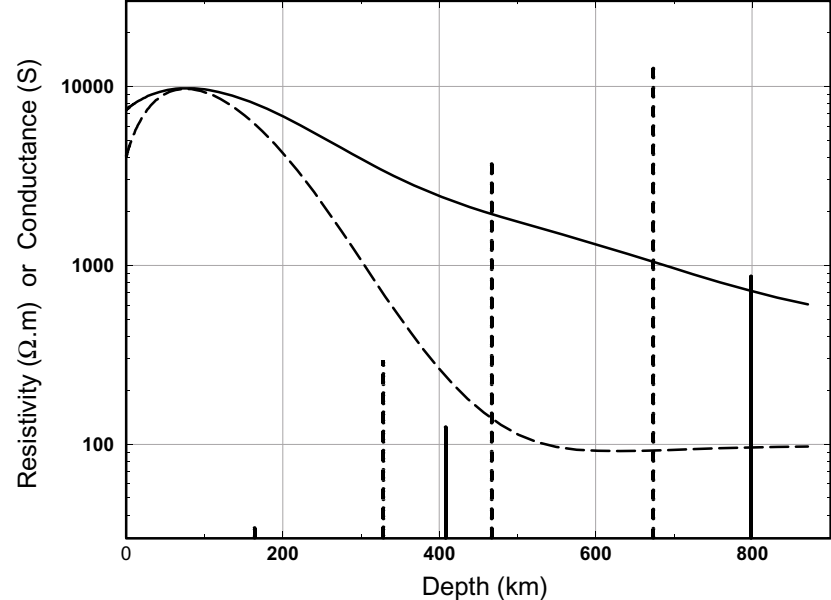

Fig. 11. Models obtained from inverting the averaged responses of the data shown in Fig. 8. Dashed lines are models obtained from the all-data responses, and full lines are the models obtained from the low activity interval responses. The vertical bars represent the conductance spike $D^{+}$models, and the smooth models are those obtained using Occam inversion.

vere oscillations. Also shown in Fig. 11 are the conductance spikes (dashed lines) of the best-fitting possible model from Parker's $D^{+}$algorithm (Parker, 1980; Parker and Whaler, 1981). The $\chi^{2}$ misfit of the $D^{+}$model to the 32 data points is 23.8, which is an RMS of below one. This demonstrates that the responses are descriptive of a causal minimum phase response function, and, without other information, these models would be taken as valid descriptions of the variation of resistivity with depth below the site.

Using a factor of 1.5 times the standard deviation from the mean, data were processed taking only those time intervals of longer than 3 hours when the vertical magnetic field variations stayed within the range -90 to $+60 \mathrm{nT}$ (shown by the locations of the bars in Fig. 4 and the shaded region in Fig. 7). Almost $30 \%$ of the data lie outside these ranges and were rejected. The off-diagonal responses for those intervals are also shown in Fig. 10 (full symbols), and the best-fitting Occam model and $D^{+}$conductance spikes in Fig. 11 (full lines).

A comparison of the responses and models are also shown in Fig. 12. The theoretical responses for a source field of $3,000 \mathrm{~km}$ wavelength over a 1-D layered Earth given by the Occam model for the low activity intervals only (solid line in Fig. 11 and thick line in Fig. 12(c)) are also plotted in Figs. 12 (dashed lines in 12(a) and 12(b)). These responses describe reasonably well the estimates obtained from processing all data (pluses in Figs. 12(a) and 12(b)). Thus, the estimates obtained from processing all data can be interpreted as being contaminated by a long wavelength source.

Estimates were also derived using time intervals for other limits, namely 1 and 2 times the standard deviation of the vertical field variations, or -47 to $+30 \mathrm{nT}$ and -115 to +85 $\mathrm{nT}$, rejecting $53 \%$ and $13 \%$ of the data respectively. These responses were virtually identical to those shown in Fig. 10 (full symbols) for the -90 to $+60 \mathrm{nT}$ limits, except that for the -47 to $+30 \mathrm{nT}$ case the time segments used are insufficiently long to determine estimates at periods greater than 

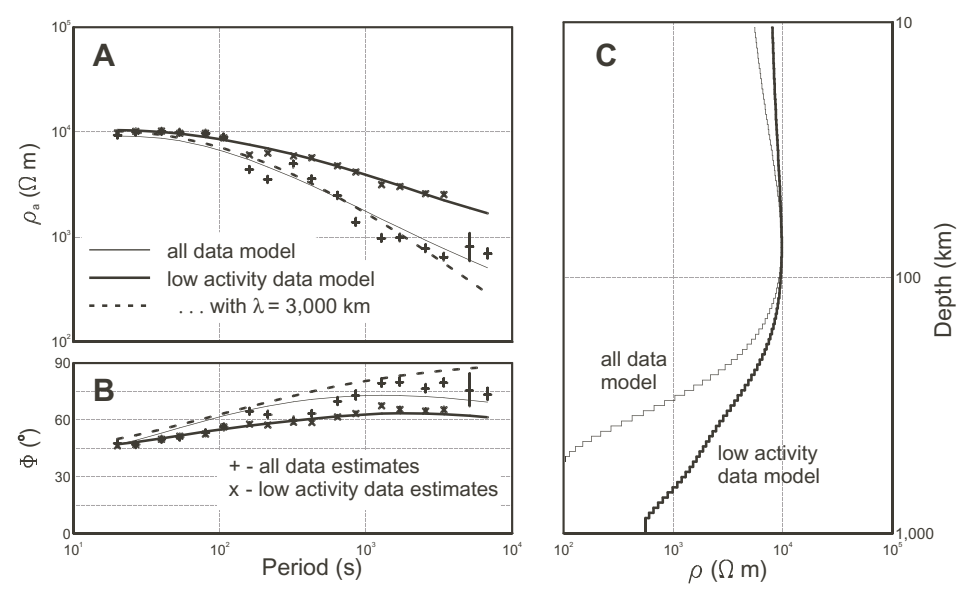

Fig. 12. Comparison of the averaged MT responses and their models; (A) Apparent resistivity data and model responses, (B) Phase data and model responses, (C) Models. The plus symbols are the responses obtained using all data, whereas the crosses are those from selected low activity intervals only. The thick lines in (A) and (B) are the responses for the thick line model in (C), and the thin lines are those of the thin line model in (C). The dashed lines are the theoretical responses for the thick line model in (C) but with a source field with a wavelength of 3,000 km. The vertical bars on the estimates in (A) and (B) indicate one sigma error estimates: where absent the errors are smaller than the symbol.

3,400 s. However, using broader limits the responses begin to be affected by source effects and to exhibit decaying apparent resistivity curves and rising phase curves. Empirically, we have found that limits of less than twice the standard deviation of the distribution yield consistent response estimates, whereas greater limits result in response estimates that display source effects to varying degree. However, this rule is dependent on activity level and should not be taken as correct in all cases.

Clearly, the robust method of Jones and Jödicke (1984) was unable to remove non-uniform source field contributions when all data were included. In other cases robust codes have been successful at reducing the effects of auroral sources, e.g., Garcia et al. (1997). However, as noted by Egbert (1997) one cannot rely upon robust methods removing coherent noise, which essentially is what the nonuniform source field effects are, and Egbert et al. (2000) demonstrate that robust processing yields estimates biased by non-uniform source fields in the 10-100 s period band.

\section{Conclusions}

The possibility of erroneous interpretation due to nonuniform source field effects in magnetotelluric (MT) responses has been discussed since the inception of the MT method. Theoretical responses for sources of various geometries have been derived, but these sources do not describe the reality of the complex interactions of the threedimensional (3-D) moving ionospheric and magnetospheric currents with the conducting Earth that occur. Hermance (1978) demonstrates that moving sources tend to effectively increase source wavelengths and thereby to decrease nonuniform effects in the data. However, this result should not be used as an excuse to ignore the possibility of contamination; particularly for high latitude sites located close to auroral zones and for low latitude sites within the influence of the equatorial electrojet.

This paper describes a simple method for determining time intervals where source contamination is likely to be low. It is based on the vertical magnetic field variations, which are far more sensitive to non-uniform source effects than are MT response functions, and is best applicable for regions that are dominantly one-dimensional (1-D), but also could be used in 2-D and 3-D regions. Intervals are selected when the vertical field variations lie within predefined limits, and these limits are chosen based on a histogram of the variations. The limits are set to a value less than twice the standard deviation of the distribution of the variations, and the responses are derived from the low activity data.

An example of application of the method is shown for a site picked specifically for its location and for its time of recording. The site was located directly beneath the auroral oval to show the effects most readily, and acquisition occurred during the lowest solar activity possible in the last 11year solar cycle. Processing all the available data results in response functions that visually seem reasonable, are physically consistent, and that can be inverted for a model that apparently reveals a sharp decrease in resistivity at around 350 $\mathrm{km}$. This model feature could be erroneously interpreted as evidence to support the one to two orders of magnitude decrease in resistivity expected at the $410 \mathrm{~km}$ phase boundary based on laboratory measurements (Xu et al., 1998).

Rejecting $30 \%$ of the high activity data and processing only data from low activity intervals results in response functions that have higher apparent resistivities and lower phases, as predicted from simple finite wavenumber arguments. The model that fits these responses does not show evidence for a low resistivity layer in the upper mantle, and indeed is quite anomalously resistive to deep within the mantle, contrary to the results of $\mathrm{Xu}$ et al. (1998). The theoretical responses for this model with an assumed 3,000 km wavelength repetitive source reasonably well describe the contaminated responses obtained from processing all of the data.

Robust methods can work well to reduce the effects of non-uniform sources, as demonstrated by Garcia et al. (1997). However, these methods can fail, as shown above, 
and the caution of Egbert (1997) regarding biases from coherence noise signals should be well heeded.

Finally, these data were taken from a time of exceptionally low sunspot activity to show that problems exist even in low activity data. The cost of using this approach is that one must reject contaminated data, and in this case some $30 \%$ are not used. For normal sunspot conditions one might expect that this number would be higher. However, the cost of not using some method of rejecting contaminated data is a contaminated model, clearly something that will lead to erroneous conclusions about Earth structure and will ultimately discredit the EM induction field.

Acknowledgments. We thank Dr. Juanjo Ledo, Mr. Jim Craven, and the two anonymous referees for their comments on an earlier version of this paper. Geological Survey of Canada Contribution Number 2001069.

\section{References}

Akasofu, S.-L., Polar and Magnetospheric Substorms, 280 pp., Springer, New York, 1968.

Cagniard, L., Basic theory of the magneto-telluric method of geophysical prospecting, Geophysics, 18, 605-635, 1953.

Camfield, P. A., Magnetometer array study in a tectonically active region of Quebec, Canada, Geophys. J. R. astr. Soc., 65, 553-570, 1981.

Constable, S. C., R. L. Parker, and C. G. Constable, Occam's inversion: a practical algorithm for generating smooth models from electromagnetic sounding data, Geophysics, 52, 289-300, 1987.

Dmitriev, V. I. and M. N. Berdichevsky, The fundamental model of magnetotelluric sounding, Proc. IEEE, 67, 1034-1044, 1979.

Egbert, G. D., Robust multiple-station magnetotelluric data processing, Geophys. J. Int., 130, 475-496, 1997.

Egbert, G. D., M. Eisel, O. S. Boyd, and H. F. Morrison, DC trains and Pc3s: Source effects in mid-latitude geomagnetic transfer functions, Geophys. Res. Lett., 27, 25-28, 2000.

Gamble, T. D., W. M. Goubau, and J. Clarke, Magnetotellurics with a remote reference, Geophysics, 44, 53-68, 1979.

Garcia, X., A. D. Chave, and A. G. Jones, Robust processing of magnetotelluric data from the auroral zone, J. Geomag. Geoelectr., 49, 1451-1468, 1997.

Hermance, J. F., Electromagnetic induction in the Earth by a moving ionospheric current system, Geophys. J. R. astr. Soc., 55, 557-576, 1978.

Hermance, J. F. and W. R. Peltier, Magnetotelluric fields of a line current, J. Geophys. Res., 75, 3351-3356, 1970.

Hibbs, R. D. and F. W. Jones, The calculation of the electromagnetic fields of a sheet current with arbitrary intensity distribution over a layered half space-I: The general method and results, Geophys. J. R. astr. Soc., 46, 433-452, 1976a.

Hibbs, R. D. and F. W. Jones, The calculation of the electromagnetic fields of a sheet current with arbitrary intensity distribution over a layered half space-II: The computer program and its application, Geophys. J. R. astr. Soc., 46, 453-465, 1976 b.

Jones, A. G., Geomagnetic induction studies in Scandinavia-I. Determination of the inductive response function from the magnetometer data, $J$. Geophys., 48, 181-194, 1980.

Jones, A. G. and I. J. Ferguson, The electric Moho, Nature, 409, 331-333, 2001.

Jones, A. G. and H. Jödicke, Magnetotelluric transfer function estimation improvement by a coherence-based rejection technique, 54th Society of Exploration Geophysics Annual General Meeting, Atlanta, Georgia, U.S.A., December 2-6, Abstract volume pages 51-55, 1984.

Jones, A. G., A. D. Chave, G. Egbert, D. Auld, and K. Bahr, A comparison of techniques for magnetotelluric response function estimation, $J$.
Geophys. Res., 94, 14,201-14,213, 1989

Kuckes, A. F., Relations between electrical conductivity of a mantle and fluctuating magnetic fields, Geophys. J. R. astr. Soc., 32, 119-131, 1973a.

Kuckes, A. F., Correspondence between the magnetotelluric and field penetration depth analysis for measuring electrical conductivity, Geophys. $J$ R. astr. Soc., 32, 381-385, 1973b.

Liu, H.-P. and D. D. Kosloff, Numerical evaluation of the Hilbert transform by the Fast Fourier Transform (FFT) technique, Geophys. J. R. astr. Soc. 67, 791-799, 1981.

Mareschal, M., Source effects and the interpretation of geomagnetic sounding data at sub-auroral latitudes, Geophys. J. R. astr. Soc., 67, 125-136, 1981.

Mareschal, M., Modelling of natural sources of magnetospheric origin in the interpretation of regional induction studies: a review, Surv. Geophys. 8, 261-300, 1986.

Narod, B. B. and J. R. Bennest, Ring-core fluxgate magnetometers for use as observatory variometers, Phys. Earth Planet. Inter., 59, 23-28, 1990.

Osipova, I. L., S. C. O. Hjelt, and L. L. Vanyan, Source field problems in northern parts of the Baltic shield, Phys. Earth Planet. Inter., 53, 337342, 1989.

Parker, R. L., The inverse problem of electromagnetic induction: existence and construction of solutions based on incomplete data, J. Geophys. Res. 85, 4421-4425, 1980 .

Parker, R. L. and K. A. Whaler, Numerical methods for establishing solutions to the inverse problem of electromagnetic induction, J. Geophys. Res., 86, 9574-9584, 1981.

Peltier, W. R. and J. F. Hermance, Magnetotelluric fields of a Gaussian electrojet, Can. J. Earth Sci., 8, 338-346, 1971.

Pettersen, F., Aurora borealis-the northern lights, Way North: Earth Science, publication from Troms $\varnothing$ Museum, Univ. Troms $\varnothing, 1,1992$.

Pirjola, R. J., Modelling the electric and magnetic fields at the Earth's surface due to an auroral electrojet, J. Atmos. Solar-Terrestr. Phys., 60, 1139-1148, 1998.

Price, A. T., The theory of magnetotelluric fields when the source field is considered, J. Geophys. Res., 67, 1907-1918, 1962.

Schmucker, U., Anomalies of geomagnetic variations in the southwestern United States, Bull. Scripps Inst. Oceanogr., Univ. Calif. Press, 13, 1970

Schmucker, U. and P. Weidelt, Electromagnetic Induction in the Earth, Lecture Notes, Aarhus Univ., Denmark, 1975.

Srivastava, S. P., Method of interpretation of magneto-telluric data when source field is considered, J. Geophys. Res., 70, 945-954, 1965.

Tikhonov, A. N., On determining electric characteristics of the deep layers of the Earth's crust, Dolk. Acad. Nauk. SSSR, 73, 295-297, 1950 Reprinted in Vozoff (1986), 2-3.

Viljanen, A., R. Pirjola, and L. Häkkinen, An attempt to reduce induction source effects at high latitudes, J. Geomag. Geoelectr., 45, 817-831 1993.

Viljanen, A., R. Pirjola, and O. Amm, Magnetotelluric source effect due to 3D ionospheric current systems using the complex image method for 1D conductivity structures, Earth Planets Space, 51, 933-945, 1999.

Vozoff, K. (ed.), Magnetotelluric Methods, Soc. Expl. Geophys. Reprint Ser. No. 5, Tulsa, OK, ISBN 0-931830-36-2, 1986.

Wait, J. R., On the relationship between telluric currents and the Earth's magnetic field, Geophysics, 19, 281-289, 1954.

Weidelt, P., The inverse problem of geomagnetic induction, Z. Geophys., 38, 257-289, 1972.

Weidelt, P., Entwicklung und Erdprobung eines Verfahrens zur Inversion zweidimensionaler Leitfähigkeitsstrukturen in E-Polarisation, Habilitation thesis, Dept. Math. Natural Sciences, Univ. Göttingen, 1978.

Xu, Y., B. T. Poe, T. J. Shankland, and D. C. Rubie, Electrical conductivity of olivine, wadsleyite and ringwoodite under upper-mantle conditions, Science, 280, 1415-1418, 1998.

A. G. Jones (e-mail: ajones@nrcan.gc.ca) and J. Spratt (e-mail: jespratt@mailbox.syr.edu) 